\title{
Computational analysis of frictional drag over transverse grooved flat plates
}

\author{
Pritanshu Ranjan, Akshoy Ranjan Paul *, Ajay Pratap Singh \\ Department of Applied Mechanics, Motilal Nehru National Institute of Technology, Allahabad, INDIA \\ *Corresponding Author: e-mail: arpaul2k@yahoo.co.in, Tel +91-532-2271212, Fax.+91-532-2271200
}

\begin{abstract}
Skin-friction coefficient of turbulent boundary layer flow over a smooth-wall with transverse square grooves is investigated for four grooved-wall cases. The four grooved-wall configurations is like'd' type rough wall, which is characterized by regularly spaced two-dimensional square cavities (grooves) placed normal to the flow. This is made up of $5 \mathrm{~mm}$ square grooved-wall, where square grooves are spaced 10, 20 and 40 element widths apart in the streamwise direction. A commercial CFD code'Fluent 6.3' is used for the mean velocity and turbulence intensity calculation. Hexahedral meshing is used to mesh the domain, with the first grid point placed at a height of $0.001 \mathrm{~mm}$ till the dimensionless wall distance, $\mathrm{y}^{+}=10$ and afterwards the grid spacing was increased by aspect ratio of 1.1 to get a structured mesh. A steady state renormalized group (RNG) $k-\varepsilon$ model is used for turbulence modeling with non-equilibrium wall functions for near wall treatment. CFD code is validated against the experimental data reported by Stuardi and Ching. The skin-friction coefficient determined from the velocity profile increases sharply just downstream of the groove. This overshoot is followed by an undershoot and then relaxation back to the smooth-wall value. This behavior is observed in most grooved-wall cases. Integrating the skin-friction coefficient in the streamwise direction indicates that there is an increase in the overall drag with maximum to be $3.54 \%$ for the single groove case.
\end{abstract}

Keywords: Turbulent boundary layer, transverse groove, skin-friction, drag reduction, computational fluid dynamics (CFD).

\section{Introduction}

Nowadays there is lot of progress going in the direction of reducing frictional drag on surfaces of engineering interest which are rough in aerodynamic sense like missiles, space shuttles, aircraft, and submarine etc. as surface roughness plays an important role in heat transfer and skin friction. Irrespective of various researches done in the field of turbulent boundary layer, there are still many aspects, like flow over transverse groove, which are not fully understood till date.

Recently, friction drag reduction is gaining interest as it plays important role in aircraft and submarine fuel efficiencies and noise reduction. While a greater emphasis has been placed on riblets because of their potential drag reduction, the use of different types of surfaces should yield important information on the way a turbulent boundary layer responds to the surface. The ' $d$ ' type rough wall, which is characterized by regularly spaced two-dimensional square cavities (grooves) placed normal to the flow, is easier to fabricate than a riblet surface. For this reason, grooves are more attractive than riblets from an engineering point of view. Also, such surfaces may be more amenable to further optimization and/or additional control. For example, if they are combined with riblets, they can further reduce skin-friction drag.

The effect of square grooves on fluid dynamics is far from being understood. Conflicting results were reported in literatures. All the previous studies were done experimentally and it has been suggested that transverse square grooves, optimally sized and located, could result in a reduction in skin-friction drag. Choi and Fujisawa (1993) studied the effect of a transverse square with $w / d_{0}=0.4$ and reported a reduction in skin-friction drag of about $1 \%$ while ignoring the pressure drags on the groove walls.

This paper was presented at the $5^{\text {th }}$ National Conference on Applicable Mathematics in Wave Mechanics and Vibrations (WMVC2010) held at Kakatiya University, Warangal, India,13-15 March,2010. 
Pearson et al. (1997) deduced that there was an overshoot in skin friction coefficient just downstream of the groove followed by an undershoot and an oscillatory relaxation back to the smooth-wall value. Elavarasan et al. (1996) found an increase in friction drag of about $3-4 \%$ for a surface with grooves spaced $s / w=20$ apart. Tani et al. (1987) found that the skin-friction on a smooth surface with grooves spaced 10-40w apart was lower than that on a corresponding smooth-wall over a certain range of Reynolds number. Sutardi and Ching (1999) reported that there is an increase in $C_{f}$ over the smooth-wall value immediately downstream of the groove, followed by a decrease and a subsequent oscillatory relaxation back to the smooth-wall value.

Since previous studies illustrated contradicting results, further investigation is required to study the effects of the transverse square grooves on skin friction for a turbulent boundary layer flow. The present study investigates flow for a Reynolds number 3000 based on momentum thickness and $w / d_{0}=0.072$ with different elemental-width configuration of grooves $(s / w=10,20$, and 40).

\section{Computational Details}

The geometry for the aforementioned problem is being generated in a commercial software GAMBIT. The plate investigated in the present study is $5150 \mathrm{~mm}$ long and $25 \mathrm{~mm}$ thick and is shown in Fig. 1 . The outer domain is of $1000 \times 1000 \times 5150 \mathrm{~mm}{ }^{3}$. and is meshed as shown in Fig. 2 The first grid point is placed at a height of $0.001 \mathrm{~mm}$ till $y^{+}=10$ and after wards the grid spacing was increased by aspect ratio of 1.1. Hexahedral meshing has been used to mesh the whole domain. Velocity inlet with $5.5 \mathrm{~m} / \mathrm{s}$ and free stream turbulent intensity of $0.5 \%$ has been defined at inlet boundary and pressure outlet with zero gauge pressure is been defined at outlet boundary. No-slip condition is given at wall and plate boundaries. A steady state based renormalized group (RNG) $k-\varepsilon$ model has been used for turbulence modeling as the flow over groove is a rapidly strained flow and the RNG model has an additional term in its $\varepsilon$ equation that significantly improves the accuracy. The transport equation for (RNG) $k-\varepsilon$ model is given below, the details are found in [3].

$$
\begin{aligned}
& \frac{\partial}{\partial t}(\rho k)+\frac{\partial}{\partial x_{i}}\left(\rho k u_{i}\right)=\frac{\partial}{\partial x_{j}}\left(\alpha_{k} \mu_{e f f} \frac{\partial k}{\partial x_{j}}\right)+G_{k}+G_{b}-\rho \varepsilon-Y_{M}+S_{k} \ldots \ldots \ldots(1) \\
& \frac{\partial}{\partial t}(\rho \varepsilon)+\frac{\partial}{\partial x_{i}}\left(\rho \varepsilon u_{i}\right)=\frac{\partial}{\partial x_{j}}\left(\alpha_{\varepsilon} \mu_{e f f} \frac{\partial \varepsilon}{\partial x_{j}}\right)+C_{1 \varepsilon} \frac{\varepsilon}{k}\left(G_{k}+C_{3 \varepsilon} G_{b}\right)-C_{2 \varepsilon} \rho \frac{\varepsilon^{2}}{k}-R_{\varepsilon}+S_{\varepsilon} \ldots .(2)
\end{aligned}
$$

The $R_{\varepsilon}$ in the equation of $\varepsilon$ transport equation is the extra term which accounts for the strained flows.

Non-equilibrium wall functions for near wall treatment is used since it is specially designed for high Reynolds number and it also accounts for pressure gradient effects even with less three-dimensional flows and, further allows non-equilibrium separation and reattachment. It employs the two-layer concept in computing the budget of turbulence kinetic energy at the walladjacent cells, which is needed to solve the $k$ equation at the wall-neighboring cells. The wall-neighboring cells are assumed to consist of a viscous sublayer and a fully turbulent layer. It effectively relaxes the local equilibrium assumption (production = dissipation) that is adopted by the standard wall function in computing the budget of the turbulence kinetic energy at wallneighboring cells [3].

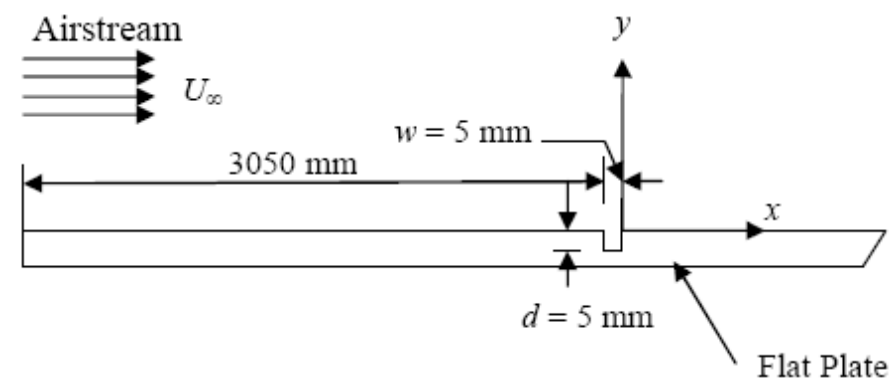

Figure 1. Schematic diagram of the grooved wall.

An implicit solution scheme is used in combination with an algebraic multigrid method to achieve faster convergence. The second-order upwind scheme is used in the discretization scheme for all equation to achieve higher accuracy in results. Velocity-pressure coupling is established by pressure-velocity correlation using a SIMPLE (Semi-Implicit Method for PressureLinked Equations) algorithm. Residuals are continuously monitored for continuity, $x$-velocity, $y$-velocity, $z$-velocity, $k$, and $\varepsilon$. Convergence of the solution is assumed when the values of all residuals goes below $10^{-6}$. 


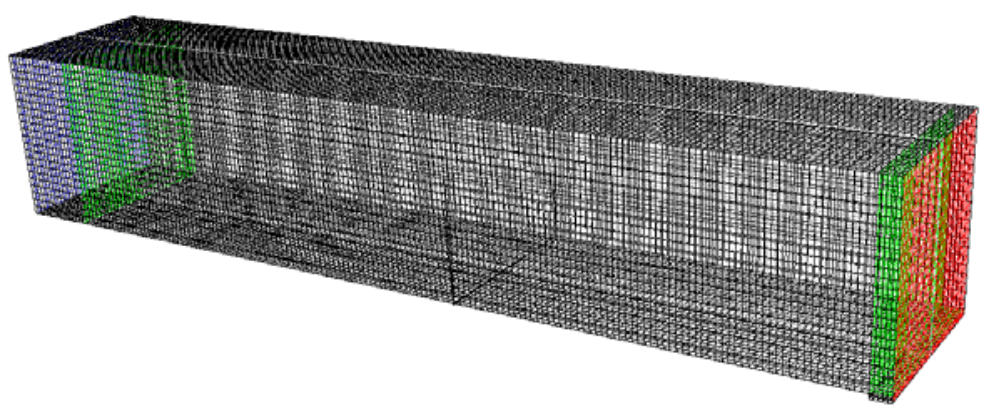

Figure 2. Meshed domain of a single grooved plate.

\section{Code Validation}

Preliminary investigations are done using a range of turbulence models to validate the CFD code with the experimental data obtained by Sutardi and Ching (1999). Experiments were performed at Reynolds number 24500 based on boundary layer thickness of $69 \mathrm{~mm}$ at $x / w=1$, so to ensure the accurate prediction of flow filed Initial calculation was done to calculate the inlet velocity profile so as to get the same boundary layer thickness $\left(d_{0}\right)$ at $x / w=1$.

To indicate the turbulence quantities, like the turbulence kinetic energy $(k)$ and the turbulence dissipation rate $(\varepsilon)$ at the inlet initially, the following relation is used:

$$
k=\frac{3}{2}\left(U_{a v i} I\right)^{2} \text { and } \varepsilon=C_{\mu}^{3 / 4} \frac{k^{3 / 2}}{L}
$$

where, $L=$ turbulence length scale $=0.07 L_{c}$

$L_{c}=$ characteristic length

$I=$ turbulence intensity $=0.16(\mathrm{Re})^{-1 / 8}$

The turbulence intensity is kept below $0.5 \%$ at the inlet.

The comparison of computed values of skin-friction coefficient $\left(C_{f_{s}}\right)$ for a smooth wall with the experimental one is shown in

Fig. 3. The figure clearly indicates a very good matching between the computed and experimental value in both value and trend wise. The average value of skin friction coefficient computed is 0.003094 as compared to 0.003181 found experimentally. The initial deviation can be attributed to the fact that in experiment a sand paper and a thin circular rod is been used to trip the boundary layer due to which the local turbulence is induced in the viscosity affected region which lowers the value of skin friction coefficient at the leading edge of the plate. Form figure it can be said that the computational model therefore under predicts the skin friction coefficient only by $2.735 \%$, which shows that the model captures the flow phenomenon reasonably well.

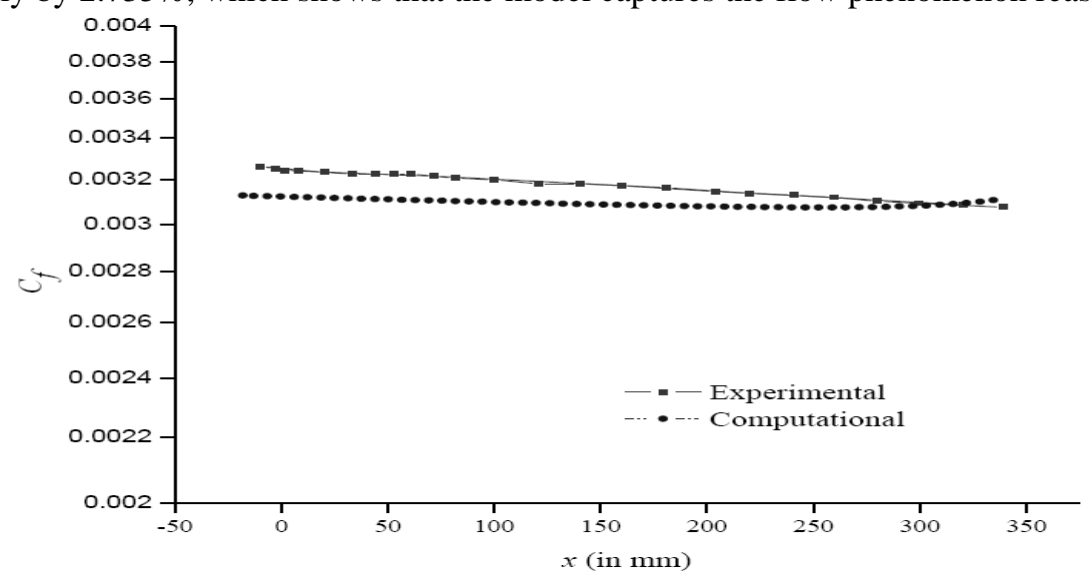

Figure 3. Comparison of experimental and computational values of skin-friction coefficient

\section{Results and Discussion}

After validating the CFD code, it is used to compute the flow parameters for different groove configuration as shown in Fig. 4. The geometry of different configuration with the geometrical details is shown below. 


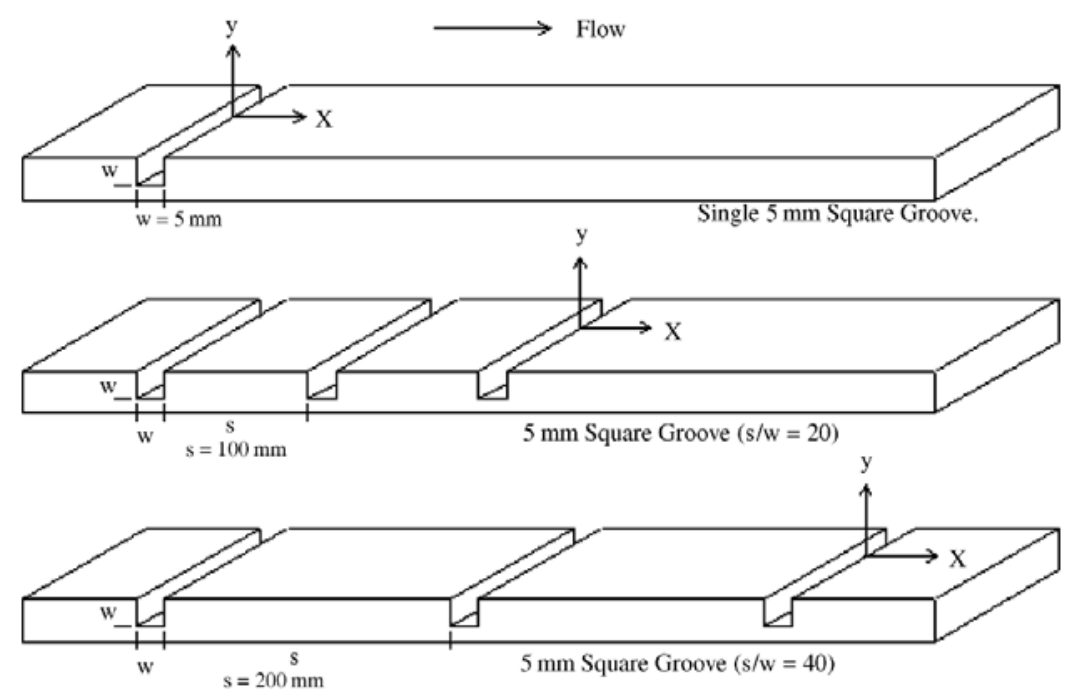

Figure 4. Different grooved wall configuration

Three geometries are investigated here:

- Single grooved plate,

- Grooves with elemental width ratio $(s / w)$ of 20 , and

- Grooves with elemental width ratio $(s / w)$ of 40.

In the first grooved-wall case, the normalized skin-friction coefficient $\left(C_{f} / C_{f_{s}}\right)$ distribution in the downstream of the groove is presented in Fig. 4. It shows a sharp rise (5.3\%) in $\left(C_{f} / C_{f s}\right)$ immediately downstream of the groove. This overshoot can be attributed to the locally intensive favorable pressure gradient that emanates from the downstream stagnation edge of the groove. The sharp rise is followed by a gradual decrease in $\left(C_{f} / C_{f s}\right)$ below the smooth-wall value in the region. The minimum $C_{f}$ is $1.91 \%$ lower than smooth-wall value. The decrease in skin-friction coefficient is then followed by an oscillatory relaxation back to the smooth-wall value. The undershoot is attributed to the fact that the flow tries to recover from the favorable pressure gradient immediately downstream of the groove to a constant freestream section in a rather small distance. This abrupt change causes the skin-friction to respond at a slower rate to the change in the boundary condition. The result is a considerable under-prediction of the skin-friction coefficient.



Figure 5. Skin-friction distribution for single groove case. 
The normalized skin-friction coefficient $\left(C_{f} / C_{f s}\right)$ distributions downstream of the groove are presented in Figs.5 and 6 for the second $(s / w=20)$ and third $(s / w=40)$ grooved-wall cases, respectively. These figures show the same qualitative $\left(C_{f} / C_{f_{s}}\right)$ behavior as in the single grooved wall case. The sharp rise in $\left(C_{f} / C_{f_{s}}\right)$ occurred just downstream of the groove in the two cases. The maximum $C_{f}$ for the second and third cases is $3.12 \%$ and $4.35 \%$ higher than $C_{f}$ of the smooth-wall, respectively, as compared with $5.3 \%$ in the single groove case. The minimum $C_{f}$ in the second and third cases are $0.88 \%$ and $1.68 \%$ less than $C_{f}$ of the smooth-wall, respectively, and $1.91 \%$ in the single groove case.

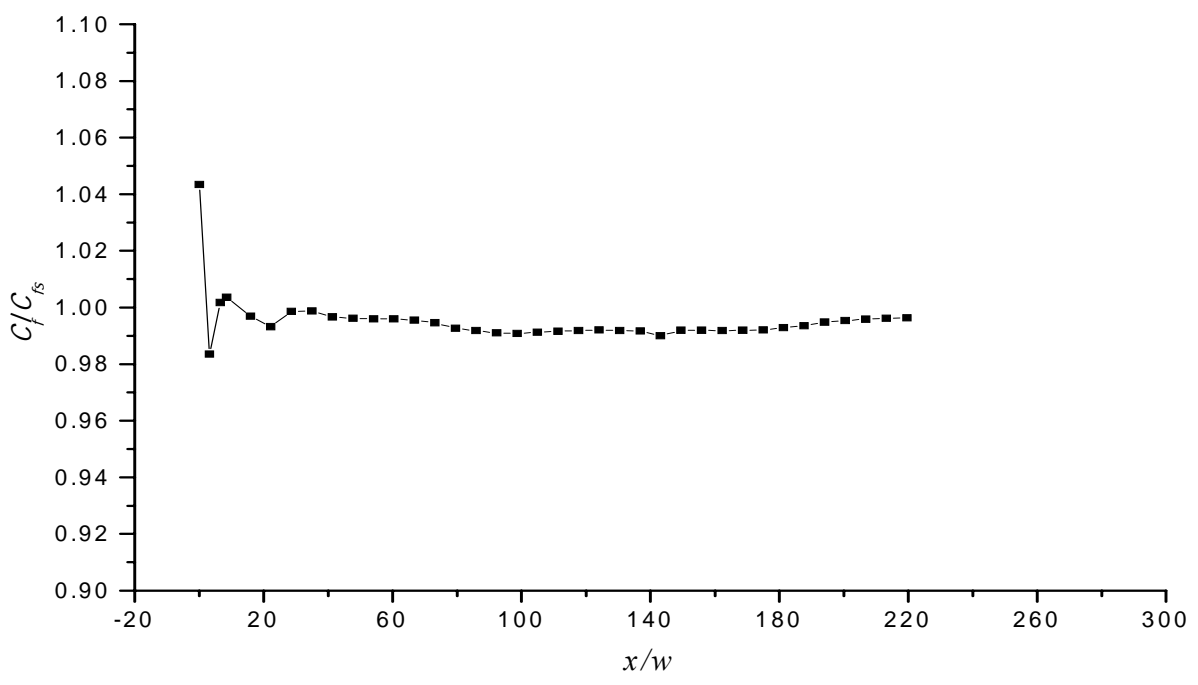

Figure 6. Skin-friction distribution for multiple $5 \mathrm{~mm}$ square grooved wall $(s / w=40)$

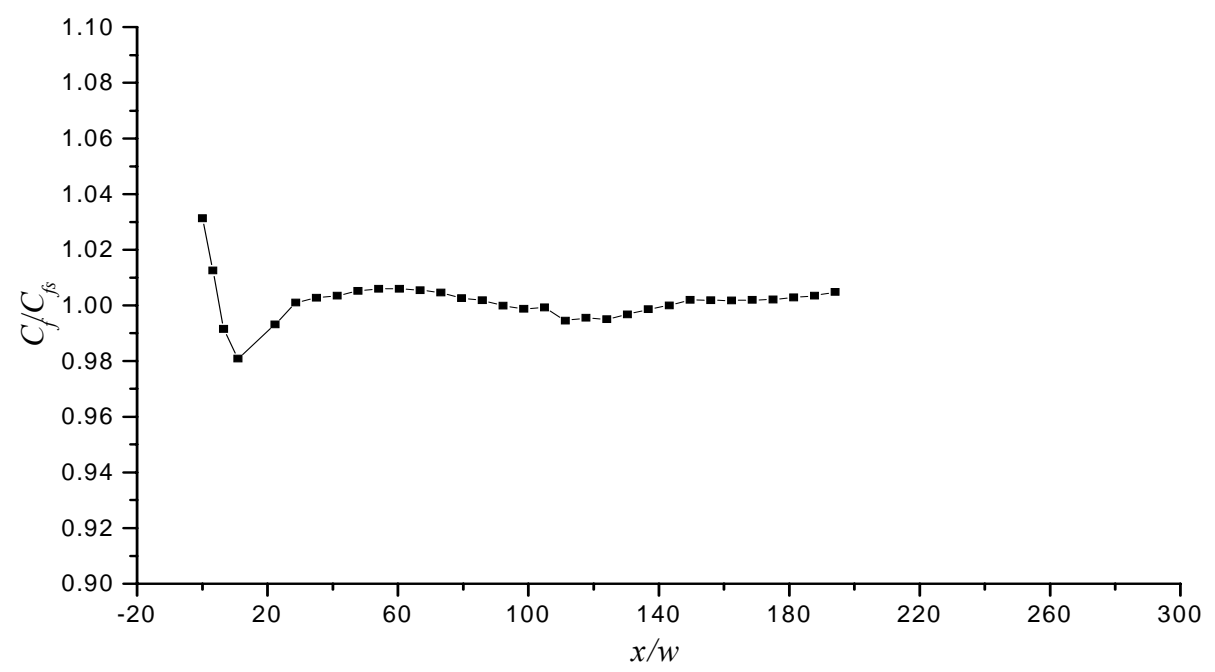

Figure 7. Skin-friction distribution for multiple $5 \mathrm{~mm}$ square grooved wall $(s / w=20)$

The reason for the differences in the intensity of the overshoots and undershoots is credited to the spacing between the grooves. The spacing downstream of the grooves affects the intensity of the overshoot and undershoots. In the third case $(s / w=40)$, the spacing between the grooves is large for the flow to relax before it reaches the downstream groove. When the flow passes the last groove, the skinfriction coefficient overshoots to $4.35 \%$ higher the smooth-wall value as compared to $5.3 \%$ in the single groove 
case and undershoots to $1.68 \%$ lower than the smooth-wall value as compared to $1.91 \%$ in the single groove case. The difference in the overshoot and undershoot intensities between the single groove case and the third grooved-wall case are $21.8 \%$ and $13.5 \%$, respectively. Closer spacing as in the second grooved-wall case $(s / w=20)$ reduces the overall effect of the grooves. Since the spacing in this case is not sufficient for the flow to relax, the flow reaches the last groove with less energy caused by the undershoot from the upstream groove. This less energy can result in reducing the intensity of the overshoot and undershoot. The skin-friction coefficient overshoot and undershoot values in the second case are $3.12 \%$ higher than the smooth-wall value and $0.88 \%$ lower than the smooth-wall value, respectively, as compared with $5.3 \%$ and $1.91 \%$ in the single groove case. The oscillatory behavior of $\left(C_{f} / C_{f s}\right)$ is less pronounced in the second case than that of the single groove case. In the third case, however, this oscillatory behavior is negligible. The drag on the first, second and third grooved-wall cases are 3.54, 3.48\% and $1.9 \%$ higher than that on the smooth-wall, respectively. The drag on the second case is very close to that of the first case because the skin-friction coefficient does not return back to that of the smooth-wall downstream of the undershoot. In the third case, however, the skin-friction coefficient rapidly recovers to that of the smooth-wall. Table 1 summarizes the drag results obtained for all grooved-wall cases.

Table 1. Summary of the drag results for various grooved wall cases

\begin{tabular}{llc}
\hline S.No. & Grooved cases & Drag reduction (\%) \\
\hline 1 & Single grooved- wall case & 3.54 \\
\hline 2 & Multiple $5 \mathrm{~mm}$ square groove, $s / w=20$ & 3.48 \\
\hline 3 & Multiple $5 \mathrm{~mm}$ square groove, $s / w=40$ & 1.90 \\
\hline
\end{tabular}

\section{Conclusions}

A computational study is done here to determine the drag coefficient on flat plate as well as plates with transverse grooves of various configurations. The findings are presented below:

- An increase in the drag over the smooth-wall is found in all the cases presented here. The maximum increase in drag (3.54\%) was found in the single grooved-wall, while the increase in drag in the second grooved-wall case $(s / w=20)$ was $3.48 \%$. The lowest increase in drag $(1.9 \%)$ was found in the third grooved wall case $(s / w=40)$.

- The spacing between the grooves $(s)$ is the determining factors for the intensity of overshoot and undershoot of $C_{f}$, the location of the $C_{f}$ undershoot, and the relaxation distance downstream of the groove.

\section{Nomenclature}

$C_{f} \quad$ skin-friction coefficient for grooved wall, $C_{f}=\tau_{w} / 0.5 \rho U_{\infty}^{2}$, dimensionless

$C_{f s} \quad$ skin-friction coefficient for smooth wall, dimensionless

$d \quad$ depth of a grove, $\mathrm{mm}$

$d_{0} \quad$ boundary layer thickness just upstream of first groove, $\mathrm{mm}$

$k \quad$ turbulent kinetic energy, $\mathrm{m}^{2} / \mathrm{s}^{2}$

$s \quad$ spacing between two consecutive grooves, $\mathrm{mm}$

$u_{*} \quad$ friction velocity at the nearest wall, $u_{*}=\sqrt{\tau_{w} / \rho}, \mathrm{m} / \mathrm{s}$

$U_{\infty} \quad$ freestream velocity of air, $\mathrm{mm}$

w width of a grove, $\mathrm{mm}$

$x \quad$ flow direction, $\mathrm{mm}$

$y \quad$ distance to the nearest wall (normal to flow direction), $\mathrm{mm}$

$y^{+} \quad$ dimensionless wall distance, $y^{+}=u_{*} y / v$

$\varepsilon \quad$ turbulent kinetic energy dissipation rate, $\mathrm{m}^{2} / \mathrm{s}^{3}$

$v \quad$ kinematic viscosity of fluid, $\mathrm{m}^{2} / \mathrm{s}$

$\rho \quad$ density of fluid, $\mathrm{kg} / \mathrm{m}^{3}$

$\tau_{w} \quad$ wall shear stress, $\mathrm{N} / \mathrm{m}^{2}$ 


\section{Acknowledgement}

The authors acknowledge the Department of Science \& Technology, Govt. of India for providing necessary funds through DSTFIST grant to carry out this research.

\section{References}

1. Choi, K., S. and Fujisawa, N., Possibility of drag reduction using d-type roughness, Appl. Sci. Res., Vol. 50, 1993, pp. 315-324.

2. Elavarasan, R., Ching, C., Y. and Antonia, R., A., Turbulent boundary layer over a smooth wall with widely separated transverse square cavities, Appl. Sci. Res., Vol. 55, 1996, pp. 227-243.

3. Fluent 6.3 user’s guide, CFD software package, Fluent Inc., Lebanon, NH03766, 2006.

4. Pearson, B., R., Elavarasan, R. and Antonia, R., A., The response of a turbulent boundary layer to a square groove, ASME J. Fluids Engg., Vol. 119, 1997, pp. 466-469.

5. Stuardi and Ching, C.Y., Effect of a transverse square groove on a turbulent boundary layer, Exp. Thermal and Fluid Science, Vol. 20, 1999, pp. 1 - 10.

6. Tani, I., Munakata, H., Matsumoto, A. and Abe, K., Turbulence management by groove roughness, In: H.W. Liepmann, R. Narasimha (Eds.), Turbulence Management and Relaminarisation, IUTAM Symposium, Bangalore, India, 1987, pp. 161-172.

\section{Biographical notes}

Pritanshu Ranjan is presently working as a visiting faculty member in Applied Mechanics department at Motilal Nehru National Institute of Technology, Allahabad, India. He did his M.Tech. in Fluids Engineering from the same department. He is involved in the flow control research group in the institute. He has published a few research papers in national and international conferences and journals.

Akshoy Ranjan Paul is an Assistant Professor in the Department of Applied Mechanics, Motilal Nehru National Institute of Technology, Allahabad, India. He has received M. E. degree from Jadavpur University , Kolkata, India in 2002. He has more than nine years of experience in teaching and research. His current research area includes internal flow aerodynamics, flow control and turbulence modeling. He has published three textbooks and many technical papers in national and international conferences and journals. He visited a few universities in UK, Denmark, and Sweden for academic visit and technical training.

Ajay Pratap Singh has recently completed his M.Tech. in Fluids Engineering from Applied Mechanics department at Motilal Nehru National Institute of Technology, Allahabad, India. He is currently working as Assistant Professor in the Mechanical Engineering department at A.K. Garg Institute of Technology, Ghaziabad, India. He has published a couple of research papers in national and international conferences.

Received September 2010

Accepted March 2011

Final acceptance in revised form March 2011 